\title{
Scaling Theory for Migration-Driven Aggregate Growth
}

\author{
F. Leyvraz \\ Centro de Ciencias Físicas, Av. Universidad s/n, Col. Chamilpa, Cuernavaca, Morelos, MEXICO
}

\author{
S. Redner \\ Center for BioDynamics, Center for Polymer Studies, and Department of Physics, Boston University, Boston MA 02215
}

(November 14, 2018)

\begin{abstract}
We give a comprehensive description for the irreversible growth of aggregates by migration from small to large aggregates. For a homogeneous rate $K(i ; j)$ at which monomers migrate from aggregates of size $i$ to those of size $j$, that is, $K(a i ; a j) \sim a^{\lambda} K(i ; j)$, the mean aggregate size grows with time as $t^{1 /(2-\lambda)}$ for $\lambda<2$. The aggregate size distribution exhibits distinct regimes of behavior that are controlled by the scaling properties of the migration rate from the smallest to the largest aggregates. Our theory applies to diverse phenomena such as the distribution of city populations, late stage coarsening of non-symmetric binary systems, and models for wealth exchange.
\end{abstract}

Much attention has been devoted to understanding the irreversible growth of aggregates through binary coalescence. This general mechanism arises in diverse branches of physics, such as gelation [1], island formation in epitaxial surface growth [2], and stellar evolution [3]. By a long-term research effort, considerable understanding of this irreversible aggregation process has been achieved [4.5]. In this Letter, we focus on a different growth mechanism that appears to provide a natural description for the evolution of city populations. This is preferential evaporation from smaller aggregates and preferential condensation onto larger aggregates

There are many examples where this evaporation/condensation mechanism occurs in physics and in the social sciences. The classic physics example is the late-stage coarsening of a binary mixture in an off-critical quench below but near the coexistence curve [6, ,7]. Here the system separates into droplets of the minority phase that are embedded in a matrix of the majority phase. Subsequent growth proceeds through preferential evaporation from smaller droplets, due to the effect of surface tension, and subsequent condensation onto the larger droplets [6].

In the social sciences, it has been argued [8] that the growth of cities may be due to migration from small to large cities, as opposed to a view that emphasizes differential population growth [9]. In a spirit closer to our work, the wealth distribution of individuals was described by a kinetic asset exchange model with preferential transfer from poor to rich individuals in each transaction [10]. For generic situations, the solution to the rate equation showed that this mechanism gives very different kinetic behavior from conventional aggregation.

Motivated by these fragmentary results, we investigate a general class of migration-driven growth phenomena and show that, at large times and for large aggregate sizes, a comprehensive scaling theory can be developed with a minimum of assumptions. This theory gives both the growth rate of the typical aggregate size, as well as the asymptotics of the aggregate size distribution. Basic features of our theory agree with data on the population dynamics of U.S. cities. An important feature of our theory is that one may infer the general form of the migration rates from observations of the aggregate size distribution. For phenomena such as the city population distribution or the wealth distribution, we may thus hope to predict basic aspects of the dynamics in systems for which we have little a priori knowledge of underlying microscopic driving mechanisms.

The model that we study is defined as follows. Let aggregates $A_{j}$ be characterized only by their mass $j$, or equivalently, by the number of individuals that comprise them. These aggregates evolve according to the following irreversible reaction

$$
A_{k}+A_{l} \stackrel{K(k ; l)}{\longrightarrow} A_{k-1}+A_{l+1} \quad k \leq l .
$$

That is, a monomer (or equivalently, one person) leaves a smaller aggregate of size $k$ and joins a larger one of size $l$ with rate $K(k ; l)$. This generalizes the asset exchange model of Ref. 10, where a restricted class of reaction rates $K(k ; l)$ were considered. More generally, migration could also go from a larger to a smaller aggregate. The symmetric limit, where the migration direction does not depend on the relative sizes of the two aggregates, leads to a diffusive-like kinetic universality class. We defer the investigation of this general system to a future work. Instead, we focus on the situation where there is preferential migration from small to large aggregates. In fact, any migration bias leads to scaling behavior for the aggregate size distribution identical to that of complete bias, as embodied by Eq. (11).

We now make the assumption of spatial homogeneity, so that the system is fully characterized by the concentrations $c_{j}(t)$ of aggregates $A_{j}$ of size $j$ at time $t$. We also assume that the law of mass-action applies so that the 
time dependence of the aggregate concentrations may be described by the following rate equations:

$\dot{c}_{j}(t)=\frac{1}{2} \sum_{k, l=1}^{\infty} K(k ; l) c_{k}(t) c_{l}(t)\left[\delta_{k, j+1}+\delta_{l, j-1}-\delta_{k, j}-\delta_{l, j}\right]$.

The various delta-function terms enforce the constraint that the size of each aggregate changes by \pm 1 in a single reaction. The initial condition may be taken to be $c_{j}(0)=\delta_{j, 1}$, but any initial condition may equally well be considered, provided it is rapidly decaying in $j$.

From these equations, we can immediately draw several important conclusions. First, there are no equilibrium solutions. Rather, the size of aggregates grows continuously and each $c_{j}(t)$ eventually goes to zero as $t \rightarrow \infty$. Second, the total mass contained in the aggregates is (formally, at least) conserved. That is,

$$
\frac{d}{d t} \sum_{j=1}^{\infty} j c_{j}(t)=0
$$

if the necessary interchanges between the infinite sums in this equation can be justified. Here we shall confine ourselves to this mass-conserving case. For definiteness we normalize the total mass to unity.

We now make the conventional scaling ansatz for the large-time behavior of $c_{j}(t)$ [5]. We assume that there exists a well-defined typical aggregate size $s(t)$ at time $t$ such that

$$
c_{j}(t) \rightarrow j^{-2} \Phi(j / s(t)) .
$$

Here the exponent -2 follows directly from the condition that the total mass is conserved, as discussed, for example, in 沺. We further assume that the reaction rates $K(k ; l)$ are homogeneous of degree $\lambda$, or at least, that they are asymptotically so in the limit of large sizes. That is

$$
K(a k ; a l)=a^{\lambda} K(k ; l)[1+o(1)] .
$$

In the context of city population growth, the homogeneity exponent $\lambda$ can be given the following interpretation. When the populations of two cities are scaled by some factor, there are both more susceptible migrants in the smaller city and potentially more reasons to move to the larger city. It is then natural that the overall migration rate varies as a power law in this scale factor. Exceptions to this behavior typically involve the existence of a cutoff size that separates two qualitatively different kinds of behaviors.

Substituting the scaling ansatz (4) into Eq. (2), we find that $s(t)$ satisfies $\dot{s}(t)=s(t)^{\lambda-1}$, with asymptotic solution, for $\lambda<2$,

$$
s(t) \propto[(2-\lambda) t]^{1 /(2-\lambda)} .
$$

Defining $z$ as the growth exponent of $s(t)$, we thus have

$$
z=\frac{1}{2-\lambda}
$$

This growth exponent can also be obtained by adaptation of a back-of-the-envelope estimate for the typical size in irreversible aggregation. In aggregation, the reaction of aggregates of typical size $s$ leads to a growth $\Delta s$ of the order of $s$ in a time $\Delta t$ of the order of $1 /\left(\right.$ concentration $\left.\times s^{-\lambda}\right)$. Here the concentration scales as $1 / s$ and $s^{-\lambda}$ is the inverse reaction rate between typical-size aggregates. This leads to $\dot{s} \sim s^{\lambda}$, from which $s \sim t^{1 /(1-\lambda)}$. For migration-driven growth, $\Delta s$ is now of the order of 1 in the time $\Delta t$. This gives $\dot{s} \sim s^{\lambda-1}$, thus reproducing the growth exponent of Eq. (7).

On the other hand, if $\lambda>2$, a power-law decay of the $c_{j}(t)$ in $j$ sets in at finite time. This feature invalidates the mass conservation statement and hence the scaling form of Eq. (位). The limiting case $\lambda=2$ can be treated within our scaling formulation, but must be handled with particular care, as we discuss below. This pattern of behavior for the time dependence of the typical size parallels that of conventional aggregation, except that the size exponent in aggregation is $z=1 /(1-\lambda)$ and a finite-time gelation transition occurs for $\lambda>1$ [1], 1 . Note also that when the migration rate is symmetric, a scaling analysis similar in spirit to that just presented shows that the mean aggregate size grows as $t^{1 /(3-\lambda)}$ for $\lambda<3$. Thus even migration without population bias leads to growing aggregates, albeit at a slower rate than if a bias towards larger aggregates exists.

Also from the scaling ansatz, we find, after some nontrivial algebra, that the scaled aggregate size distribution $\Phi(x)$ obeys

$$
\frac{d \Phi(x)}{d x}=-x \frac{d}{d x}\left[\frac{\Phi(x) \Psi(x)}{x^{2}}\right],
$$

with

$$
\Psi(x)=\int_{0}^{\infty} \frac{d y}{y^{2}}[K(x ; y)-K(y ; x)] \Phi(y) .
$$

From these equations the basic qualitative behavior of $\Phi(x)$ can be deduced. Note that only the antisymmetric part of $K(x ; y)$ contributes to the scaling limit. Without loss of generality, we can now assume that $K(k ; l)=0$ for $k>l$. From Eq. (8a) it follows that $\Phi(x)$ can be discontinuous whenever $\Psi(x)+x$ becomes zero. In particular, at such a point $x_{c}, \Phi(x)$ can be consistently set to zero for all $x>x_{c}$. As long as $\Phi(x)$ is different from zero, however, (8a) can be integrated to yield

$$
\Phi(x)=\frac{A x^{2}}{x+\Psi(x)} \exp \left[-\int_{x_{0}}^{x} \frac{d y}{y+\Psi(y)}\right] .
$$

Here $A$ and $x_{0}$ are arbitrary constants chosen so that 


$$
\int_{0}^{\infty} \frac{\Phi(y)}{y} d y=\sum_{j=1}^{\infty} j c_{j}(0)=1 .
$$

An important feature of $\Phi(x)$ is its behavior for small values of $x$. To quantify this, we define the exponent $\tau$ through

$$
\Phi(x) \propto x^{2-\tau}[1+o(1)] .
$$

With this definition, one has $c_{j}(t) \sim j^{-\tau}$ for $1 \ll j \ll$ $s(t)$, as well as

$$
c_{j}(t) \sim t^{-(2-\tau) z} \equiv t^{-w} \quad(1 \ll j \ll s(t)) .
$$

This defines the exponent $w$.

To proceed further, we introduce another fundamental exponent that completes the scaling characterization of the reaction rates $K(k ; l)$, namely,

$$
K(1 ; l) \approx l^{\lambda-\mu} \quad(l \rightarrow \infty) .
$$

This is entirely analogous to the corresponding definition in conventional aggregation where the form of the cluster size distribution depends on the relative rates of small-small, large-large, and large-small reactions [ $[$ ]. With these definitions, we find, after detailed analysis of Eq. (9), four different classes of behavior:

- Type 1: $\lambda \geq 1, \mu>1$. In this case $\tau=\lambda$ and hence $w=1$.

- Marginal: $\lambda>1, \mu=1$. Here it is not possible to make simple statements about the value of $\tau$. Rather, $\tau$ depends on the complete shape of $\Phi(x)$ and therefore on the very specific form of the reaction rates.

- Type 2a: $\lambda<1, \mu \leq(1+\lambda) / 2$. In this case $\tau=\mu$ and $w=(2-\mu) /(2-\lambda)$.

- Type 2b: $\lambda<1,(1+\lambda) / 2 \leq \mu<1$. In this case $\tau=(1+\lambda) / 2$ and $w=(3-\lambda) /(4-2 \lambda)$.

For the complementary large- $x$ behavior of $\Phi(x)$, we now show that in almost all cases $\Phi(x)$ vanishes beyond a certain critical value $x_{c}$ of its argument. Indeed, suppose the contrary. It then follows from Eq. (8b) that $\Psi(x) \rightarrow-\infty$ as $x \rightarrow \infty$. This can happen in three ways: either $\Psi(x)$ varies faster than linear, slower than linear, or linearly in $x$. In the first case, Eq. (9) would indicate that $\Phi(x)<0$ for large $x$, which is impossible. In the second case, $\Phi(x)$ would go to a constant as $x \rightarrow \infty$, in contradiction to Eq. (10). Thus the only viable possibility is the third case, which occurs if $\lambda-\mu=1$.

A more thorough investigation is required to determine whether it is possible to find a consistent large- $x$ behavior for $\Phi(x)$ in this last case. If so, then $\Phi(x)$ would have a power-law decay such that the integral in Eq. (10) still converges. However, in all other cases, Eq. (9) must cease to be valid at some point and the function $\Phi(x)$ must vanish identically afterwards. This can happen in two different ways: Either $x+\Psi(x)$ has a simple zero at some point $x_{c}$ and the function $\Phi(x)$ jumps from its value at $x_{c}$ to zero, which is possible according to Eq. (8a), or else the function $\Phi(x)$ goes smoothly to zero at $x_{c}$ as a consequence of Eq. (9) and the double zero of $x+\Psi(x)$ at $x_{c}$. These results closely correspond to those of the Lifshitz-Slyozov-Wagner (LSW) theory of coarsening [6], as well as to models of asset exchange [10].

A special case that can be solved exactly is the case $\mu=\lambda$. For this situation, we find

$$
\begin{aligned}
& \Phi(x)=x^{2-\lambda} \quad\left(x \leq(2-\lambda)^{1 /(2-\lambda)}\right) \\
& \Phi(x)=0 \quad \text { otherwise }
\end{aligned}
$$

If $\lambda>1$, this case belongs to a system of Type 1 listed above, whereas if $\lambda<1$, this case belongs to Type 2a. For either alternative, the correct exponent $\tau$ is predicted. Note further the discontinuity in $\Phi(x)$ that indeed occurs exactly at the point where $x+\Psi(x)$ vanishes. Many other cases can be handled similarly and will be presented in a forthcoming publication [11].

A situation that requires a more refined analysis is $\lambda=2$ and $\mu>1$. For these parameter values, it follows that $\tau=2$, which is incompatible with the normalization condition Eq. (10). To obtain valid results, we need to modify the scaling ansatz as follows

$$
c_{j}(t) \approx \frac{j^{-2}}{\ln s(t)} \Phi(j / s(t))
$$

It follows that

$$
s(t)=\exp [\sqrt{2(t+B)}],
$$

where $B$ is some constant. The function $\Phi(x)$ then has the normalization $\Phi(0)=1$ and satisfies a modified version of Eq. (8a).

Let us now discuss how our scaling theory applies to LSW coarsening [6]. For this system, the migration rate $K(i ; j)$ is given by the product of the rate at which a particle evaporates from an aggregate of mass $i$ and the probability that it reaches an aggregate of size $j$. In the evaporation step, the diffusive current $J$ is $\Delta c / R(i)$, where $\Delta c$ is the difference between the monomer concentration near the interface and in the bulk. This difference is proportional to $R(i)^{-1}$, since it is due to surface tension. The current $J$ is therefore of the order of $R(i)^{-2}$ and thus the rate at which particles leave an aggregate of size $i$ is proportional to $J R(i)^{d-1}$, that is, to $R(i)^{d-3}$. Further, the probability of reaching an aggregate of size $j$ in three dimensions is simply proportional to its volume $R(j)^{d}$. We therefore find for the overall migration rate

$$
K(i ; j) \approx R(i)^{d-3} R(j)^{d} \approx i^{(d-3) / d} j .
$$

From the definitions of $\lambda$ and $\mu$ in Eqs. (5) and (13), it follows that the system is of Type $2 \mathrm{a}$, from which one 
obtains $z=d / 3$ and $\tau=1-3 / d$. These indeed correspond to the LSW predictions, in which the characteristic cluster radius increases as $\left.t^{1 / 3} \| 6\right]$ and the number $n(R)$ of clusters of radius $R \ll R_{c}(t)$ varies as $R^{2}$ [13]. Our theory also correctly predicts that the scaling function vanishes beyond a certain value of the scaling variable. On the other hand, the migration rate of Eq. (17) is not precise enough to ensure that our scaling theory reproduces the same functional form for $\Phi(x)$ as that of the detailed LSW theory.

To apply our theory meaningfully to the evolution of city populations, it is necessary to incorporate the effects of demographic population growth. Over intermediate time scales (of the order of decades), demographic growth typically gives a population that increases exponentially with time. Such a behavior can be modeled by allowing the process $A_{k} \longrightarrow A_{k+1}$ to occur at rate $k \gamma$. When demographic growth and migration occur together, the scaling ansatz for the underlying rate equations needs to be modified accordingly. We have found that the appropriate scaling ansatz for this more realistic situation is

$$
c_{j}(t) \approx j^{-2} e^{\gamma t} \Phi(j / s(t)) .
$$

With this hypothesis, the functional form of $\Phi(x)$ turns out to remain the same as the case of no demographic growth, but now the typical city population grows as

$$
s(t) \sim e^{\gamma t /(2-\lambda)} .
$$

Hence we arrive at the central conclusion that the typical city size grows much faster than the population of the country as a whole as $\lambda$ approaches 2 .

The city population distribution in many countries is consistent with a power-law form in which the exponent $\tau$ is close to 2 [8, 9, 12]. Our scaling theory then requires that the homogeneity exponent $\lambda$ is also close to 2 . Thus from Eq. (19), the typical city population should increase much faster than the overall population. This is confirmed qualitatively by data for the populations of various U.S. cities during their early histories [14]. The population of essentially every major U.S. city grows much faster than the U.S. as a whole over considerable time range. However, as cities reach maturity, their growth may slow or their population may even decline for reasons unrelated to preferential migration to still larger cities.

In summary, we have introduced a simple kinetic description for migration-driven growth and developed a scaling theory that determines the large-time behavior for the aggregate size distribution. Asymptotic results depend only on rudimentary properties of the reaction rates, most notably the homogeneity index $\lambda$. The typical aggregate size grows as $t^{1 /(2-\lambda)}$, while several distinct behaviors emerge for aggregate size distribution. Our results represent the counterpart of the scaling theory of irreversible aggregation to migration-driven growth. Finally, we have suggested a connection between migrationdriven growth to the distribution of city populations and found a qualitative correspondence between model predictions and recent data on U.S. cities.

We are grateful to grants NSF INT9600232, NSF DMR9978902, and DGAPA IN112200 for financial support of this work.

[1] For a survey of the rate equation for aggregation and its application to gelation, see, e.g., R. L. Drake, in Topics in Current Aerosol Research, eds. G. M. Hidy and J. R. Brock, Vol. III, part 2, (Pergamon, Oxford, U. K., 1972). See also R. M. Ziff, E. M. Hendricks, and M. H. Ernst, Phys. Rev. Lett. 49, 593 (1982).

[2] A. Pimpinelli and J. Villain, Physics of Crystal Growth (Cambridge University Press, U.K., 1998).

[3] See e.g., S. Chandrasekhar, Rev. Mod. Phys. 151 (1943); G. B. Field and W. C. Saslaw, Astrophys. J. 142, 568 (1965).

[4] P. G. J. van Dongen and M. H. Ernst, Phys. Rev. Lett. 54, 1396 (1985).

[5] M. H. Ernst, in: Fundamental Problems in Statistical Physics VI, ed. E. G. D. Cohen (Elsevier, New York, 1985).

[6] I. M. Lifshitz and V. V. Slyozov, J. Phys. Chem. Solids 19, 35 (1961); C. Wagner, Z. Elektrochemie, 65, 581 (1961).

[7] A. J. Bray, Adv. Phys. 43, 357 (1994).

[8] M. Marsili and Y. C. Zhang, Phys. Rev. Lett. 80, 2741 (1998).

[9] D. H. Zanette and S. C. Manrubia, Phys. Rev. Lett. 79, 523 (1997).

[10] S. Ispolatov, P. L. Krapivsky and S. Redner, Eur. Phys. J. B 2, 267 (1998). For related work, see A. Dragulescu and V. M. Yakovenko, Eur. Phys. J. B 17, 723 (2000).

[11] F. Leyvraz and S. Redner, to be published

[12] G. K. Zipf, Human Behavior and the Principle of Least Effort Addison-Wesley Press, Inc., Cambridge (1949).

[13] Note that the equivalence between the number $n(R)$ of clusters of a given radius and the number $c_{j}(t)$ of clusters of a given mass $j$ is given by $c_{j}(t) \approx R(j)^{-(d-1)} n[R(j)]$.

[14] Population data is available at the U.S. census bureau website: http://www.census.gov. City population data by year is available at http://physics.bu.edu/ redner. 